\title{
Emergent Interacting Spin Islands in a Depleted Strong-Leg Heisenberg Ladder
}

\author{
D. Schmidiger, ${ }^{1, *}$ K. Yu. Povarov, ${ }^{1}$ S. Galeski, ${ }^{1}$ N. Reynolds, ${ }^{1, \dagger}$ R. Bewley ${ }^{2}$ T. Guidi, ${ }^{2}$ J. Ollivier, ${ }^{3}$ and A. Zheludev ${ }^{1, \$}$ \\ ${ }^{1}$ Neutron Scattering and Magnetism, Laboratory for Solid State Physics, ETH Zürich, CH-8093 Zürich, Switzerland \\ ${ }^{2}$ ISIS Facility, Rutherford Appleton Laboratory, Chilton, Didcot, Oxon OX11 0QX, United Kingdom \\ ${ }^{3}$ Institut Laue-Langevin, 6 rue Jules Horowitz, 38042 Grenoble, France
}

(Received 1 March 2016; published 21 June 2016)

\begin{abstract}
Properties of the depleted Heisenberg spin ladder material series $\left(\mathrm{C}_{7} \mathrm{H}_{10} \mathrm{~N}\right)_{2} \mathrm{Cu}_{1-z} \mathrm{Zn}_{z} \mathrm{Br}_{4}$ have been studied by the combination of magnetic measurements and neutron spectroscopy. Disorder-induced degrees of freedom lead to a specific magnetic response, described in terms of emergent strongly interacting "spin island" objects. The structure and dynamics of the spin islands is studied by highresolution inelastic neutron scattering. This allows us to determine their spatial shape and to observe their mutual interactions, manifested by strong spectral in-gap contributions.
\end{abstract}

DOI: 10.1103/PhysRevLett.116.257203

In the solid state, even weak perturbations may lead to qualitatively new physics described in terms of entirely new emergent degrees of freedom and quasiparticles [1]. One such perturbation, known to open the door to a variety of novel and competing ground states and rich phase diagrams, is structural or chemical disorder [2]. An exciting example of disorder-induced emergent degrees of freedom are the magnetic objects that appear upon the introduction of nonmagnetic impurities in gapped quantum-disordered antiferromagnets (AFs) [3-10]. These entities may be understood as spins released from nonmagnetic AF singlets by removing their partner spins. The short-range correlations in the underlying quantum AFs spread these spin degrees of freedom over extended regions ("spin islands") around each impurity site [3]. The size of the spin islands is controlled by the correlation length in the parent system, and may be as large as dozens of nanometers. Because of their partial overlap, these spin islands interact. The original quantum AF thus acts as a "medium" that hosts a new magnetic system of mesoscopic objects and carries interactions between them. Because of these interactions, the emergent system may have its own unique correlations and dynamics.

The impurity-induced formation of localized $S=1 / 2$ spin objects has been thoroughly studied in gapped $S=1$ Haldane spin chains [4,11-15]. Unfortunately, in these highly one-dimensional chain materials, each impurity completely severs the host system. The emergent spin islands located at the chain segment ends merely pair up into isolated dimers [4], and have no collective dynamics. This is in contrast to expectations for Heisenberg spin ladders, which are composed of two neighboring chains linked by rung interactions $J_{\perp}$. Within this topology, the nonmagnetic impurities have a low chance to disrupt the continuity of the system and a finite-size segment typically contains a large number of mutually interacting islands. To date, the study of nonmagnetic impurities in spin ladders was limited to rather complex materials with prohibitively large energy scales, such as $\mathrm{Sr}\left(\mathrm{Cu}_{1-z} \mathrm{Zn}_{z}\right)_{2} \mathrm{O}_{3}[7,16]$ or $\mathrm{Bi}\left(\mathrm{Cu}_{1-z} \mathrm{Zn}_{z}\right)_{2} \mathrm{PO}_{6}$ [5]. These cases are difficult to describe theoretically, as they involve additional interactions such as cyclic exchange, frustration, or $3 \mathrm{D}$ coupling. What is missing is an experimental study of emergent and interacting spin islands in a clean realization of the spin ladder model, such that could be compared to theoretical calculations at the quantitative level. Fortunately, in recent years, a number of new, exceptionally good organic spin ladder compounds were discovered [17]. Among them, $\left(\mathrm{C}_{7} \mathrm{H}_{10} \mathrm{~N}\right)_{2} \mathrm{CuBr}_{4}$ (DIMPY) $[18,19]$ realizes the rare case of a strong-leg spin ladder, which is of special interest in the context of the present study. Its dominant leg interactions imply a significant correlation length and hence significant interactions between emerging spin objects, even at low concentration. In the present Letter we show that DIMPY, diluted with nonmagnetic zinc, is indeed a perfect prototype for testing this physics. By combining thermodynamic and high-resolution inelastic neutron scattering experiments with numerical simulations, we are able to directly study the shape and interactions of the spin islands, and to quantitatively describe the impurity-induced collective response in the language of these emergent objects.

The parent material DIMPY was shown to be described by a simple Heisenberg spin-ladder Hamiltonian [20] with $J_{\|}=1.42(6) \mathrm{meV}$ along the leg and $J_{\perp}=0.82(2) \mathrm{meV}$ (see Fig. 1) [19,21-23] and with exceptionally weak additional interactions [24-26]. We were able to chemically introduce nonmagnetic impurities by replacing a fraction $z$ of magnetic $S=1 / 2 \mathrm{Cu}^{2+}$ by nonmagnetic $\mathrm{Zn}^{2+}$ ions [27]. The magnetic susceptibility and isothermal magnetization data are presented in Fig. 2, as measured on a series of $\left(\mathrm{C}_{7} \mathrm{H}_{10} \mathrm{~N}\right)_{2} \mathrm{Cu}_{1-z} \mathrm{Zn}_{z} \mathrm{Br}_{4}$ single crystals with varying impurity concentration $z$ [20]. While the susceptibility of the $z=0$ parent compound is being dominated by a spectral gap of $\Delta=0.33 \mathrm{meV}$ and exponentially decays to zero at 

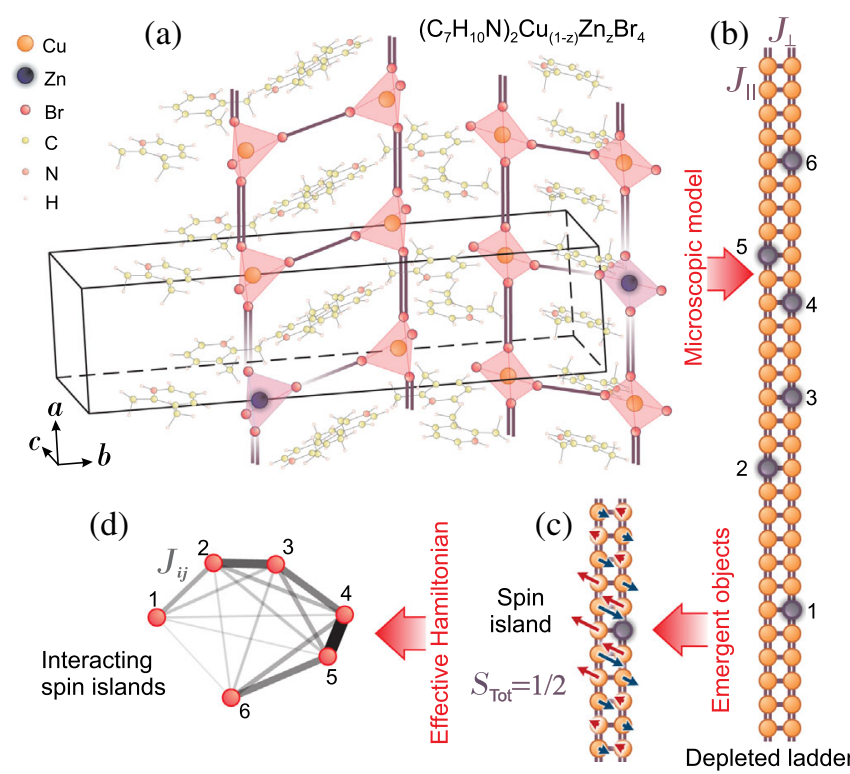

FIG. 1. From a real material to the microscopic model and then to the effective description in terms of emergent objects. (a) The crystal structure of $\left(\mathrm{C}_{7} \mathrm{H}_{10} \mathrm{~N}\right)_{2} \mathrm{Cu}_{1-z} \mathrm{Zn}_{z} \mathrm{Br}_{4}$. (b) Spin ladder model microscopic description. The random replacement of magnetic $\mathrm{Cu}^{2+}$ by nonmagnetic $\mathrm{Zn}^{2+}$ renders some $S=1 / 2$ sites missing. (c) Emerging local spin degrees of freedom (spin islands), pinned to the impurity position but with a magnetization profile extending over many unit cells. (d) Effective spin island Hamiltonian (1) with interactions controlled by the mutual distances as given by Eq. (2).

low temperatures, the susceptibility of the Zn-diluted material acquires an additional contribution, progressively increasing with the impurity concentration. This behavior becomes even more apparent in the low-temperature magnetization [Fig. 2(b)]. The latter remains suppressed by the gap below the critical field $H_{c} \simeq 2.7 \mathrm{~T}$ [19] in the clean material but acquires an impurity-induced contribution in the Zn-substituted derivatives. Nonetheless, the observed response is not simply described by the $S=1 / 2$ Brillouin function, as it would in the case of free magnetic moments [28], thus proving the importance of interactions between emergent spin degrees of freedom. While deviations from the Brillouin function are small at low concentration $z$, they become up to $45 \%$ at $z=0.06$. Qualitatively, this is explained by the mean impurity distance $\bar{L}_{x}(z)=$ $(1-z) /\left(2 z-z^{2}\right)$ [29], which is as large as 50 unit cells for $z=0.01$ but becomes comparable to the correlation length of $\xi \approx 6.3$ unit cell lengths $a$ [20] at $z=0.06$. The probability of finding close and strongly interacting islands thus rapidly increases with impurity concentration.

As a first step, we compare our measurements to numerical calculations [20] based on the full parent spin ladder Hamiltonian [19], depleted with randomly placed nonmagnetic sites [Fig. 1(b)]. Following Ref. [29], we calculate the susceptibility and magnetization with quantum Monte Carlo (QMC) simulations [30] of ladder
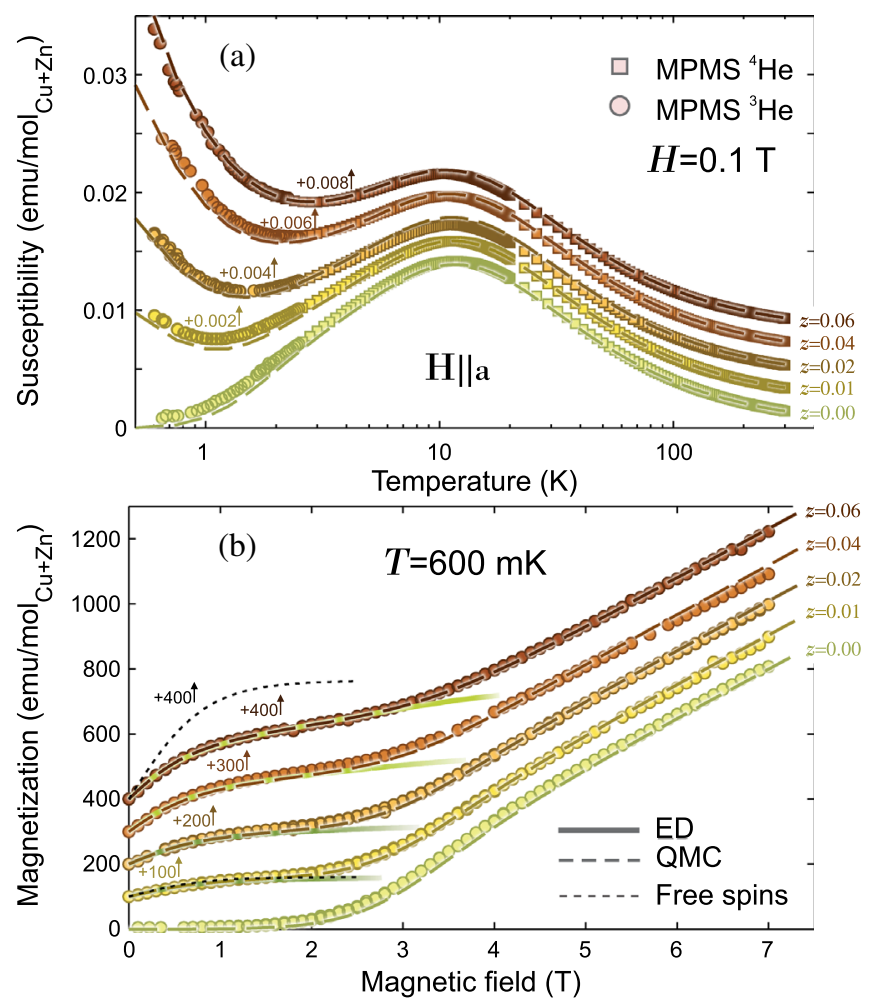

FIG. 2. Magnetization experiments on the depleted spin-ladder series $\left(\mathrm{C}_{7} \mathrm{H}_{10} \mathrm{~N}\right)_{2} \mathrm{Cu}_{1-z} \mathrm{Zn}_{z} \mathrm{Br}_{4}$. (a) Susceptibility as a function of temperature. (b) Isothermal magnetization at $T=600 \mathrm{mK}$ for the same samples. Symbols are the experimental data [20]. Numerical QMC simulations and results from the diagonalization of the effective model [Eq. (1)] are shown as dashed and solid lines, respectively. Dotted lines in (b) illustrate the free-spin response for $z=0.01$ and $z=0.06$. For clarity, data for different $z$ were vertically shifted as indicated by arrows and the corresponding shift values.

systems with $L=500$ rungs, $N=2 L z$ randomly placed nonmagnetic sites, and averaged over 300 random impurity configurations [31]. Remarkably, the numeric results (dashed lines in Fig. 2) quantitatively reproduce the measured data in the entire temperature and field range. This proves not only that we are able to chemically control the zinc concentration but also that its introduction does not lead to significant local distortion effects altering the superexchange interactions.

Extensive QMC calculations of large depleted systems, averaged over hundreds of configurations are expensive and time consuming. An alternative, much simpler approach is provided by the effective low-energy description in terms of interacting spin islands [Figs. 1(c), 1(d)] $[3,8,29]$. The latter are represented by $S=1 / 2$ spins $\mathbf{S}_{\mathbf{I}}$ at random impurity positions $\mathbf{I}$, interacting with distancedependent effective interactions $J^{\text {eff }}(\mathbf{L})$ and described by the Hamiltonian [29]

$$
\mathcal{H}_{\text {spin island }}=\sum_{\mathbf{I}, \mathbf{J}} J^{\mathrm{eff}}(\mathbf{I}-\mathbf{J}) \mathbf{S}_{\mathbf{I}} \cdot \mathbf{S}_{\mathbf{J}}-g \mu_{B} H \sum_{\mathbf{I}} S_{\mathbf{I}}^{z},
$$


with $\mu_{B}$ and $g$ being the Bohr magneton and the electron $g$ factor. Mutual spin-island interactions are mediated by the spin correlations in the hosting ladder medium. Since the latter are short-ranged, effective interactions exponentially decay with distance. In addition, due to their antiferromagnetic nature, $J^{\text {eff }}(\mathbf{L})$ are ferro- or antiferromagnetic, depending on whether the mutual interaction path contains an odd or even number of sites [3,29], reminiscent of the oscillatory Ruderman-Kittel-Kasuya-Yosida (RKKY) interaction [32]. Being fully controlled by the spin ladder medium, the effective interactions can be numerically obtained from a system with two nonmagnetic sites at distance $\mathbf{L}$ in a long ladder system. Following Refs. [3,29] we have performed matrix product state calculations $[31,33]$ and confirmed that $J^{\text {eff }}(\mathbf{L})$ is described by the simple law

$$
J^{\mathrm{eff}}(\mathbf{L})=J_{0}(-1)^{L_{x}+L_{y}+1} e^{-\left|L_{x}\right| / \xi},
$$

with the energy scale $J_{0}=0.441 \mathrm{meV}$, the decay length of $\xi=6.28$ unit cell lengths $a$ for the exchange constants of DIMPY [20]. By including the numerically calculated effective interactions, Eq. (1) becomes a parameter-free description of the emergent spin island system.

The magnetization and susceptibility of the effective model were determined [20] by exactly diagonalizing (ED) the Hamiltonian in Eq. (1), for a system of $N=12$ sites $S_{\mathrm{I}}$, randomly placed on a ladder with $L=N / 2 z$ rungs and with the effective interactions given by Eq. (2). The magnetization, averaged over 5000 random configurations, is in excellent agreement with measured data [Fig. 2(b)]. Notably, a model described by not more than 12 mutually interacting spin operators is sufficient to model the entire thermodynamic response of this complex disordered manybody quantum system. Nevertheless, the description in terms of Eq. (1) remains valid only as long as the mutual interactions controlled by the spin island correlations do not change. While the latter remain unaffected by temperature and applied field as long as the spin ladder remains in its quantum disordered regime, they fundamentally change once the system crosses the critical field $H_{c} \simeq 2.7 \mathrm{~T}$ [19] to the quantum critical Tomonaga-Luttinger spin liquid (TLSL) state. Above $H_{c}$, the effective description naturally fails.

So far, we have successfully described the measured thermodynamic properties in terms of an effective model of the emergent spin islands. However, can such objects and their interactions be observed directly? To answer this question, we present a series of high-resolution inelastic neutron scattering experiments enabling us to access the spectral properties of both the spin ladder medium and the low-energy spin island system. In Figs. 3(a) and 3(c), we show the background-subtracted magnetic neutron spectrum of $\left(\mathrm{C}_{7} \mathrm{D}_{10} \mathrm{~N}\right)_{2} \mathrm{Cu}_{0.96} \mathrm{Zn}_{0.04} \mathrm{Br}_{4}$, as measured with the neutron time-of-flight (TOF) technique (LET spectrometer
[34], ISIS facility, UK) and obtained with the Horace software [35]. Data were gathered in distinct low- and high-resolution setups with incident energies $E_{i}=4.2$ and $2.2 \mathrm{meV}(T=75 \mathrm{mK})$. Neutron intensity is shown as a function of energy transfer $\hbar \omega$ and momentum transfer $Q_{\|}=\mathbf{Q} \cdot \mathbf{a}$ along the leg direction. Corresponding data from the parent compound is shown in Figs. 3(b) and 3(d), measured under identical experimental conditions [23] and treated in the same way.

First, we observe that the properties of the spin ladder medium hosting the spin island system remains nearly unaffected by the presence of impurities [Figs. 3(a) and 3(b)]. In both the parent and disordered compound, identical gapped and dispersive magnon and two-magnon bound state branches [19] are observed. In contrast, clear changes are found in the low-energy sector Figs. 3(c) and 3(d): the magnon gap is slightly shifted from $\Delta=$ 0.33 (3) to $0.41(2) \mathrm{meV}$, reminiscent of the magnon blueshift observed in 1D quantum-disordered AFs at finite temperature [36]. However, as a main feature, we observe a stripe of enhanced in-gap intensity developing around
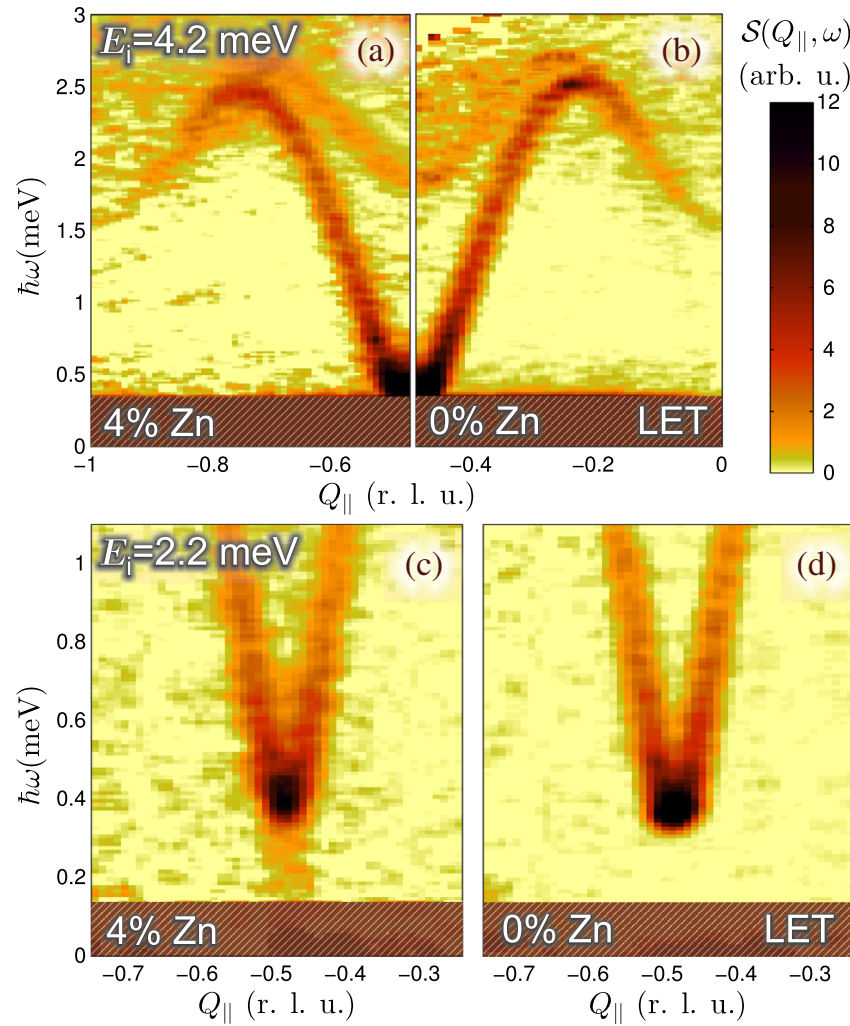

FIG. 3. Inelastic neutron scattering data on the depleted and clean spin ladder DIMPY, measured on the LET instrument. (a), (b) Entire spectrum in the lower resolution mode (incident energy $E_{i}=4.2 \mathrm{meV}$ ). (c),(d) Low-energy sector in the higher resolution mode $\left(E_{i}=2.2 \mathrm{meV}\right)$. Background corrected magnetic neutron scattering intensity is shown as a function of energy transfer $\hbar \omega$ and momentum transfer along the leg $Q_{\|}$. Hatched regions are contaminated by parasitic nuclear incoherent scattering. 
$Q_{\|}=0.5$ r.l.u. in the disordered compound. This contribution originates in strongly interacting spin islands, leading to a disorder-induced finite density of states inside the gap.

It is even more pronounced in the spectrum presented in Fig. 4(a), measured with a lower incident energy of $E_{i}=$ $1.3 \mathrm{meV}$ and thus better resolution (IN5 instrument [37], ILL, France). The spin island contribution progressively increases with decreasing energy $\hbar \omega$, as shown in Fig. 4(b). This is readily explained since at $z=0.04$, the probability of finding spin islands only weakly interacting with others remains large and there is a vast number of quantum states at low energies.

As a main characteristic, these in-gap contributions exhibit an intrinsic $Q_{\|}$linewidth, as shown in Fig. 4(c). This intrinsic linewidth is a measure of the spin island real-space magnetization profile. Similar to conventional magnetic neutron scattering [38], where intensity is modulated by the magnetic form factor (the Fourier transform of the magnetic ions'unpaired electron density), scattering by spin islands is modulated by the square of the spin island form factor $F\left(Q_{\|}, Q_{\perp}\right)$-the Fourier transform of its real space shape. A similar reasoning was applied to determine the spatial shape of emergent local objects in the substantially different cases of the chargedoped or depleted Haldane spin chains $\mathrm{Y}_{2-z} \mathrm{Ca}_{z} \mathrm{BaNiO}_{5}$ [39], $\mathrm{Y}_{2} \mathrm{BaNi}_{1-z} \mathrm{Mg}_{z} \mathrm{O}_{5}$ [4]. For a spin island with exponentially decaying staggered magnetization on a ladder, the form factor reads as
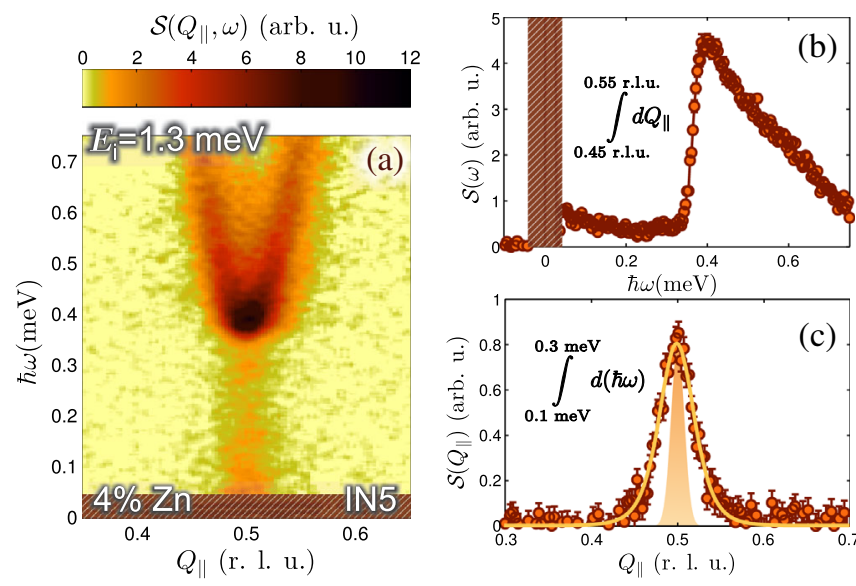

FIG. 4. Inelastic neutron scattering data on the disordered DIMPY, measured on the IN5 instrument. (a) Background subtracted spectrum measured with $E_{i}=1.3 \mathrm{meV}$. Neutron intensity is shown as a function of energy transfer $\hbar \omega$ and momentum transfer $Q_{\|}$. (b) Neutron intensity as a function of $\hbar \omega$ for $Q_{\|}$between 0.45 and 0.55 r.l.u. Data in the hatched area is contaminated by parasitic nuclear incoherent scattering. (c) Scattering intensity as a function of $Q_{\|}$, after integrating in $0.1 \mathrm{meV} \leq \hbar \omega \leq 0.3 \mathrm{meV}$. Instrumental resolution (shaded area) is shown along with the resolution-convoluted fit (3) [solid line].

$$
F\left(Q_{\|}, Q_{\perp}\right)=\frac{\left(1-e^{-i 2 \pi Q_{\perp}}\right) \sinh \left(\xi^{-1}\right)}{\cos \left(2 \pi Q_{\|}\right)+\cosh \left(\xi^{-1}\right)}-1
$$

Here, $\xi$ denotes the real space decay length and $Q_{\perp}$ is the momentum transfer along the rung [40]. Because of the dominant $\mathrm{AF}$ leg exchange and the, hence, slowly decaying and alternating magnetization profile, the form factor is peaked at $Q_{\|}=0.5$ r.l.u. with an intrinsic width much smaller than the reciprocal lattice unit. We compared $\left|F\left(Q_{\|}, Q_{\perp}\right)\right|^{2}$, averaged over $Q_{\perp}$, to our experimental data and by convolution with experimental resolution, we were able to quantify the real space decay length to be $\xi=5.0(2)$ unit cell lengths $a$. The magnetization profile thus decays to $10 \%$ of its initial value only after 15 unit cells and the size of an emergent spin island is of the order of $20 \mathrm{~nm}$. Notably, the determined decay length is slightly shorter than the numerically calculated value of $\xi=6.27$ [20]. However, this is not surprising since in-gap states observed in experiments are located at a comparatively large energy transfer on the order of $0.1 \mathrm{meV}$ and thus mainly originate from dimers or clusters of spin islands close to each other. Influenced by the nearby spin islands, the magnetization profile thus deviates from one of an isolated spin island.

In conclusion, by combining thermodynamic and highresolution inelastic neutron scattering techniques with numerical simulations, we were able to quantitatively study the properties of a system of strongly interacting spin islands formed in a strong-leg Heisenberg spin ladder depleted with nonmagnetic impurities. An effective description in the language of these objects explains and quantitatively reproduces the measured data. As long as the spin-ladder medium remains quantum disordered at $H<H_{c}$, this effective description is faithful and, in principle, would not only allow us to describe the thermodynamic response but even to access dynamical quantities such as time-dependent correlation functions [41]. Beyond $H_{c}$, however, the effective description breaks down due to the quantum phase transition to the TLSL state [42], rendering the spin-ladder correlation functions fundamentally different. To our knowledge, the problem of nonmagnetic impurities and the fate of spin islands in the TLSL phase of a spin ladder or their influence on the magnetically ordered state $[19,43]$ has not been addressed experimentally or theoretically. We hope that our study stimulates further efforts and experiments towards understanding the impurity-induced physics in such systems.

This work is partially supported by the Swiss National Science Foundation, Division II. We would like to thank Dr. S. Mühlbauer (FRM II, Technische Universität München) for his involvement at the early stages of this project and Dr. S. Gvasaliya (ETH Zürich) for assistance with the magnetic measurements. Simulations were performed on the Brutus cluster (ETH Zürich). 
*schmdavi@phys.ethz.ch

${ }^{\dagger}$ Present address: Laboratory for Neutron Scattering, Paul Scherrer Institut, CH-5232 Villigen, Switzerland.

*zhelud@ethz.ch; http://www.neutron.ethz.ch/

[1] P. W. Anderson, More is different, Science 177, 393 (1972).

[2] E. Dagotto, Complexity in strongly correlated electronic systems, Science 309, 257 (2005).

[3] H.-J. Mikeska, U. Neugebauer, and U. Schollwöck, Spin ladders with nonmagnetic impurities, Phys. Rev. B 55, 2955 (1997).

[4] M. Kenzelmann, G. Xu, I. A. Zaliznyak, C. Broholm, J. F. DiTusa, G. Aeppli, T. Ito, K. Oka, and H. Takagi, Structure of End States for a Haldane Spin Chain, Phys. Rev. Lett. 90, 087202 (2003).

[5] J. Bobroff, N. Laflorencie, L. K. Alexander, A. V. Mahajan, B. Koteswararao, and P. Mendels, Impurity-Induced Magnetic Order in Low-Dimensional Spin-Gapped Materials, Phys. Rev. Lett. 103, 047201 (2009).

[6] M. Hase, I. Terasaki, Y. Sasago, K. Uchinokura, and $\mathrm{H}$. Obara, Effects of substitution of $\mathrm{Zn}$ for $\mathrm{Cu}$ in the spin-Peierls cuprate, $\mathrm{CuGeO}_{3}$ : The suppression of the spin-Peierls transition and the occurrence of a new spinglass state, Phys. Rev. Lett. 71, 4059 (1993); M. Hase, K. Uchinokura, R. J. Birgeneau, K. Hirota, and G. Shirane, Neutron-scattering study of magnetism in single-crystal $\mathrm{Cu}_{1-x} \mathrm{Zn}_{x} \mathrm{GeO}_{3}$, J. Phys. Soc. Jpn. 65, 1392 (1996); K. Uchinokura, Spin-peierls transition in $\mathrm{CuGeO}_{3}$ and impurity-induced ordered phases in low-dimensional spin-gap systems, J. Phys. Condens. Matter 14, R195 (2002).

[7] G. B. Martins, E. Dagotto, and J. A. Riera, Rapid suppression of the spin gap in $\mathrm{Zn}$-doped $\mathrm{CuGeO}_{3}$ and $\mathrm{SrCu}_{2} \mathrm{O}_{3}$, Phys. Rev. B 54, 16032 (1996).

[8] R. Yu, T. Roscilde, and S. Haas, Field induced disorderedlocal-moment phase in site-diluted spin-gap antiferromagnets, New J. Phys. 10, 013034 (2008); R. Yu, O. Nohadani, $\mathrm{S}$. Haas, and T. Roscilde, Magnetic bose glass phases of coupled antiferromagnetic dimers with site dilution, Phys. Rev. B 82, 134437 (2010); K. Trinh, S. Haas, R. Yu, and T. Roscilde, Correlations in quantum spin ladders with site and bond dilution, Phys. Rev. B 85, 035134 (2012).

[9] S. Sahling, G. Remenyi, C. Paulsen, P. Monceau, V. Saligrama, C. Marin, A. Revcolevschi, L. P. Regnault, and J. E. Lorenzo, Experimental realization of long-distance entanglement between spins in antiferromagnetic quantum spin chains, Nat. Phys. 11, 255 (2015).

[10] T. Guidi, B. Gillon, S. A. Mason, E. Garlatti, S. Carretta, P. Santini, A. Stunault, R. Caciuffo, J. van Slageren, B. Klemke, A. Cousson, G. A. Timco, and R. E. P. Winpenny, Direct observation of finite size effects in chains of antiferromagnetically coupled spins, Nat. Commun. 6, 7061 (2015).

[11] S. H. Glarum, S. Geschwind, K. M. Lee, M. L. Kaplan, and J. Michel, Observation of fractional spin $S=1 / 2$ on open ends of $S=1$ linear antiferromagnetic chains: Nonmagnetic doping, Phys. Rev. Lett. 67, 1614 (1991).

[12] B. Batlogg, S-W. Cheong, and L. W. Rupp, Jr., Haldane spin state in $\mathrm{Y}_{2} \mathrm{Ba}(\mathrm{Ni}, \mathrm{Zn}$ or $\mathrm{Mg}) \mathrm{O}_{5}$, Physica (Amsterdam) 194-196B, 173 (1994).

[13] F. Tedoldi, R. Santachiara, and M. Horvatić, ${ }^{89}$ Y NMR Imaging of the Staggered Magnetization in the Doped
Haldane Chain $\mathrm{Y}_{2} \mathrm{BaNi}_{1-x} \mathrm{Mg}_{x} \mathrm{O}_{5}$, Phys. Rev. Lett. 83, 412 (1999).

[14] Y. Uchiyama, Y. Sasago, I. Tsukada, K. Uchinokura, A. Zheludev, T. Hayashi, N. Miura, and P. Böni, Spin-VacancyInduced Long-Range Order in a New Haldane-Gap Antiferromagnet, Phys. Rev. Lett. 83, 632 (1999).

[15] T. Masuda, K. Uchinokura, T. Hayashi, and N. Miura, Impurity-induced antiferromagnetic phase in a doped Haldane system $\mathrm{Pb}\left(\mathrm{Ni}_{1-x} \mathrm{Mg}_{x}\right)_{2} \mathrm{~V}_{2} \mathrm{O}_{8}$, Phys. Rev. B 66, 174416 (2002).

[16] M. Azuma, Y. Fujishiro, M. Takano, M. Nohara, and H. Takagi, Switching of the gapped singlet spin-liquid state to an antiferromagnetically ordered state in $\mathrm{Sr}\left(\mathrm{Cu}_{1-\mathrm{x}} \mathrm{Zn}_{\mathrm{x}}\right)_{2} \mathrm{O}_{3}$, Phys. Rev. B 55, R8658 (1997).

[17] C. P. Landee and M. M. Turnbull, Recent Developments in Low-Dimensional Copper(II) Molecular Magnets, Eur. J. Inorg. Chem. 2013, 2266 (2013).

[18] A. Shapiro, C. P. Landee, M. M. Turnbull, J. Jornet, M. Deumal, J. J. Novoa, M. A. Robb, and W. Lewis, Synthesis, Structure, and Magnetic Properties of an Antiferromagnetic Spin-Ladder Complex: Bis(2,3dimethylpyridinium) Tetrabromocuprate, J. Am. Chem. Soc. 129, 952 (2007).

[19] D. Schmidiger, P. Bouillot, S. Mühlbauer, S. Gvasaliya, C. Kollath, T. Giamarchi, and A. Zheludev, Spectral and thermodynamic properties of a strong-leg quantum spin ladder, Phys. Rev. Lett. 108, 167201 (2012).

[20] See Supplemental Material at http://link.aps.org/ supplemental/10.1103/PhysRevLett.116.257203 for detailed description of experiments and numerical calculations.

[21] D. Schmidiger, S. Mühlbauer, A. Zheludev, P. Bouillot, T. Giamarchi, C. Kollath, G. Ehlers, and A. M. Tsvelik, Symmetric and asymmetric excitations of a strong-leg quantum spin ladder, Phys. Rev. B 88, 094411 (2013).

[22] D. Schmidiger, P. Bouillot, T. Guidi, R. Bewley, C. Kollath, T. Giamarchi, and A. Zheludev, Spectrum of a magnetized strong-leg quantum spin ladder, Phys. Rev. Lett. 111, 107202 (2013).

[23] K. Yu. Povarov, D. Schmidiger, N. Reynolds, R. Bewley, and A. Zheludev, Scaling of temporal correlations in an attractive Tomonaga-Luttinger spin liquid, Phys. Rev. B 91, 020406 (2015).

[24] M. Jeong, H. Mayaffre, C. Berthier, D. Schmidiger, A. Zheludev, and M. Horvatić, Attractive Tomonaga-Luttinger Liquid in a Quantum Spin Ladder, Phys. Rev. Lett. 111, 106404 (2013); M. Jeong, D. Schmidiger, H. Mayaffre, M. Klanjšek, C. Berthier, W. Knafo, G. Ballon, B. Vignolle, S. Krämer, A. Zheludev, and M. Horvatić, Dichotomy between Attractive and Repulsive Tomonaga-Luttinger Liquids in Spin Ladders, arXiv:1604.05252.

[25] V. N. Glazkov, M. Fayzullin, Yu. Krasnikova, G. Skoblin, D. Schmidiger, S. Mühlbauer, and A. Zheludev, ESR study of the spin ladder with uniform Dzyaloshinskii-Moriya interaction, Phys. Rev. B 92, 184403 (2015).

[26] M. Ozerov, M. Maksymenko, J. Wosnitza, A. Honecker, C. P. Landee, M. M. Turnbull, S. C. Furuya, T. Giamarchi, and S. A. Zvyagin, Electron spin resonance modes in a strong-leg ladder in the Tomonaga-Luttinger liquid phase, Phys. Rev. B 92, 241113 (2015). 
[27] T. Yankova, D. Hüvonen, S. Mühlbauer, D. Schmidiger, E. Wulf, S. Zhao, A. Zheludev, T. Hong, V. O. Garlea, R. Custelcean, and G. Ehlers, Crystals for neutron scattering studies of quantum magnetism, Philos. Mag. 92, 2629 (2012).

[28] R. M. White, Quantum Theory of Magnetism, Magnetic Properties of Materials, 3rd ed. (Springer-Verlag, Berlin, 2007).

[29] A. Lavarélo, G. Roux, and N. Laflorencie, Magnetic responses of randomly depleted spin ladders, Phys. Rev. B 88, 134420 (2013).

[30] O. F. Syljuåsen and A. W. Sandvik, Quantum Monte Carlo with directed loops, Phys. Rev. E 66, 046701 (2002).

[31] B. Bauer, L. D. Carr, H. G. Evertz, A. Feiguin, J. Freire, S. Fuchs, L. Gamper, J. Gukelberger, E. Gull, S. Guertler et al., The ALPS project release 2.0: Open source software for strongly correlated systems, J. Stat. Mech. (2011) P05001.

[32] M. A. Ruderman and C. Kittel, Indirect exchange coupling of nuclear magnetic moments by conduction electrons, Phys. Rev. 96, 99 (1954); T. Kasuya, A theory of metallic ferro- and antiferromagnetism on Zener's model, Prog. Theor. Phys. 16, 45 (1956); K. Yosida, Magnetic properties of $\mathrm{Cu}-\mathrm{Mn}$ alloys, Phys. Rev. 106, 893 (1957).

[33] M. Dolfi, B. Bauer, S. Keller, A. Kosenkov, T. Ewart, A. Kantian, T. Giamarchi, and M. Troyer, Matrix product state applications for the ALPS project, Comput. Phys. Commun. 185, 3430 (2014).

[34] R. I. Bewley, J. W. Taylor, and S. M. Bennington, LET, a cold neutron multi-disk chopper spectrometer at ISIS,
Nucl. Instrum. Methods Phys. Res., Sect. A 637, 128 (2011).

[35] R. A. Ewings, A. Buts, M. D. Le, J. van Duijn, I. Bustinduy, and T. G. Perring, HORACE: software for the analysis of data from single crystal spectroscopy experiments at time-offlight neutron instruments, arXiv:1604.05895.

[36] A. Zheludev, V. O. Garlea, L.-P. Regnault, H. Manaka, A. Tsvelik, and J.-H. Chung, Extended Universal Finite- $T$ Renormalization of Excitations in a Class of OneDimensional Quantum Magnets, Phys. Rev. Lett. 100, 157204 (2008).

[37] J. Ollivier and H. Mutka, IN5 cold neutron time-of-flight spectrometer, prepared to tackle single crystal spectroscopy, J. Phys. Soc. Jpn. 80, SB003 (2011).

[38] G. L. Squires, Introduction to the Theory of Thermal Neutron Scattering (Cambridge University Press, Cambridge, England, 1978).

[39] G. Xu, G. Aeppli, M. E. Bisher, C. Broholm, J. F. DiTusa, C. D. Frost, T. Ito, K. Oka, R. L. Paul, H. Takagi, and M. M. J. Treacy, Holes in a quantum spin liquid, Science 289, 419 (2000).

[40] D. Schmidiger, S. Mühlbauer, S. N. Gvasaliya, T. Yankova, and A. Zheludev, Long-lived magnons throughout the Brillouin zone of the strong-leg spin ladder $\left(\mathrm{C}_{7} \mathrm{H}_{10} \mathrm{~N}\right)_{2} \mathrm{CuBr}_{4}$, Phys. Rev. B 84, 144421 (2011).

[41] D. Schmidiger (unpublished).

[42] T. Giamarchi, Quantum Physics in One Dimension (Oxford University Press, Oxford, 2004).

[43] T. Giamarchi, C. Rüegg, and O. Tchernyshyov, BoseEinstein condensation in magnetic insulators, Nat. Phys. 4, 198 (2008). 